\title{
Exact and approximate nonlinear waves generated by the periodic superposition of solitons
}

\author{
By John P. Boyd, Dept. of Atmospheric, Oceanic \& Space Sciences and \\ Laboratory for Scientific Computation, University of Michigan, \\ 2455 Hayward Avenue, Ann Arbor, MI 48109, USA
}

\section{Introduction}

It may be shown through the Poisson summation formula that every periodic function may be represented as an "imbricate" series [7]:

$$
u(x)=u_{0}+\sum_{n=-\infty}^{\infty} S(x+2 n \pi)
$$

where $u_{0}$ is a constant and $S(x)$ is the so-called "pattern" function, which must decay sufficiently fast as $|x| \rightarrow \infty$ so that the series converges.

If $S(x)$ is a solitary wave which is narrow in comparison to the spatial period so that adjacent copies of the soliton overlap only slightly, then one would expect that the imbricate-soliton series would be a good approximation to an exact, spatially periodic solution of the same nonlinear wave equation. What is remarkable is that Toda and others [1-5] showed that this superposition is exact even in the limit of infinitesimal amplitude when $u(x)$ is a sine wave! ${ }^{1}$

As noted in the abstract, however, all known extensions of Toda's work are limited to periodic solutions which are elliptic functions or limiting cases of elliptic functions. It is not, however, necessary that the nonlinear wave equation should be "integrable" in the sense that it may be solved by the inverse scattering method. The so-called "Regularized Long Wave" or "Benjamin-Bona-Mahony" equation is not integrable [10], but its cnoidal waves are elliptic functions and Boyd [2] has shown that these are the imbrication of the $\operatorname{sech}^{2}$ solitons.

In this note, we attempt to find a non-elliptic example by studying the quartic $K d V$ equation [6], which for waves travelling at a phase speed $c$ may be written in a coordinate system moving with the wave as

$$
u_{x x x}+4 u^{3} u_{x}-c u_{x}=0 \quad \text { ["Quartic } K d V \text { " Eq.] }
$$

This differs from the ordinary Korteweg-deVries equation only in the nonlinearity. It is a particularly instructive example because by multiplying (2) by $u_{x x}$ and integrating and then multiplying the result by $u_{x}$ and integrating, one may show that the solutions of (2) are hyperelliptic functions, that is, are the inverses of hyperelliptic integrals [8]. Since such functions are very special, one might suppose that "hypercnoidal" waves would be the likeliest candidate for a non-elliptic extension of the soliton-superposition theorems.

1 The first proof was published in 1975 [1], more than eighty years (!) after Korteweg and deVries discovered and named the cnoidal wave. 
Numerical comparison of solitons and hypercnoidal waves shows, alas, that the quartic $K d V$ periodic solutions are not the "imbrication" of the solitary wave. However, the imbricate-soliton series is a good approximation to the hypercnoidal wave. To understand the error in this approximation, a little background is needed.

The hypercnoidal waves of spatial period $2 \pi$ are a two-parameter family; we choose $\left\{c, a_{0}\right\}$ as the parameters where $c$ is the phase speed and $a_{0}$ is the coefficient of the constant in the Fourier series for $u(x)$. There is also a one-parameter family of isolated solitary waves that vanish at $x=+\infty$ plus a two-parameter family of generalized solitons that asymptote to a constant $u_{\infty}$. All solutions are symmetric about the origin and translationally invariant. Because of a dilational symmetry, one can rescale all periodic solutions to a period of $2 \pi$ without loss of generality. Proofs of these assertions are given in Boyd [12]. The dilational symmetry theorem states that if $u(x ; c)$ is a solution, then so is

$$
v\left(x ; \lambda^{2} c\right)=\lambda^{2 / 3} u(\lambda x ; c)
$$

for any positive constant $\lambda$.

\section{Numerical studies}

To test the soliton-superposition principle, we made the following sequence of calculations:

(i) Calculate the soliton $S(x)$ via the methods of Boyd [9].

(ii) Imbricate $S(x)$ as in (1) to generate a periodic function.

(iii) Substitute this imbricate-soliton series into (2) and vary the phase speed so as to minimize the integral of the differential equation residual:

$$
R(c) \equiv \int_{-\pi}^{\pi} d x\left[u_{x x x}+4 u^{3} u_{x}-c u_{x}\right]^{2}
$$

(iv) For that value of $c$ which minimized the residual, solve (2) for the hypercnoidal wave using the Fourier methods of Boyd [7]. The imbricate series is the first guess for Newton iteration.

(v) Calculate the pointwise error of the soliton-imbricate series.

Full details are given in Boyd [7, 9, 12], but the reason that step (iii) is necessary is that although the imbricate-soliton series gives the exact shape of a cnoidal wave for the Korteweg-deVries equation, the phase speed of the periodic solution is different from that of the superposed solitons. Thus, even in the Kortweg-deVries case, it is necessary to minimize the residual to determine the phase speed.

When tested against the Korteweg-deVries equation, we obtained an error of about $1 \times 10^{-7}$, consistent with the error of the numerical methods. For the hypercnoidal waves, however, there was invariably a much larger difference between the sum of the solitons and the periodic solution which most closely approximates it.

Nonetheless, the difference is small as shown in Fig. 1. For the parameters in the illustration, the amplitude is sufficiently small that the wave is well-approximated by a single cosine (plus constant). Even so, the imbricate-soliton series is a good approximation.

Further evidence for the same conclusion over a wide parameter range is offered in Table 1 . Because the quartic $K d V$ equation is primarily of theoretical interest, Table 1 is limited to $u_{\infty}=-1$. This choice allows one to see that the soliton superposition approximation is useful for generalized solitons as well as for the isolated solitary waves 


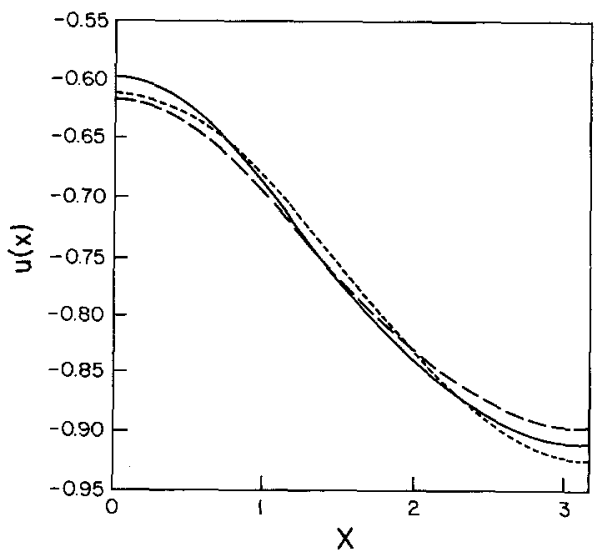

Fig. 1. A comparison of the exact hypercnoidal solution for a phase speed of $c=-2.819$ (solid) with the truncation of the hypercnoidal Fourier series to just two terms (constant plus $\cos (x)$ ) (dotted) and with the imbricate series formed from solitons such that $c_{\text {sol }}=-2.75$ and $u_{\infty}=-1$. The close agreement between the three curves implies that in this parameter regime, the periodic solution can be equally well though of as a linear cosine wave, only slightly modified by nonlinear effects, or as a sum of independent, evenly spaced and identical solitary waves, overlapping and interacting very weakly.

that vanish as $|x| \rightarrow \infty$. Experiments showed that the amplitude and width of the waves varies rapidly with $c$ but is almost independent of $u_{\infty}$. Thus, this one-dimensional slice is quite representative of the full two-dimensional parameter space.

The parameters in the table are defined as followed:

$c_{\text {sol }} \equiv$ speed of $S(x)$ [as an isolated solitary wave]

$\delta c \equiv$ correction to $c_{\text {sol }}$ which minimizes $R(c)$

$\|$ error $\|_{\infty} \equiv \max \left|u(x)-u_{\mathrm{imb}}(x)\right|$

$\alpha \equiv u_{\text {sol }}(0)-u_{\infty} \quad$ [peak-to-trough soliton amplitude]

$\mu=2 u_{\text {sol }}(\pi) / \alpha \quad$ ["relative soliton overlap"]

$v \equiv a_{1} / \sum_{n=2}^{\infty}\left|a_{n}\right| \quad$ ["first harmonic dominance parameter']

Table 1

The superposition of solitons with $u_{\infty}=-1$ for various soliton phase speeds ranging from large amplitude, narrow solitons $\left(c_{\text {sol }}=3\right)$ to short, broad solitons whose imbricate series is well-described by small-amplitude perturbation theory $\left(c_{\mathrm{sol}}=-2.75\right)$. No solutions exist for $c_{\mathrm{sol}}<-3$. The parameter $v$ is large when $u(x)$ is well-approximated by a constant plus a single cosine; $\mu$ is proportional to the (relative) overlap between neighboring solitons, and $\alpha$ is the peak-to-trough amplitude of the imbricated solitary waves.

\begin{tabular}{rrrlll}
\hline$c_{\text {sol }}$ & $\delta c$ & $v$ & $\mu$ & $\alpha$ & $\|$ error $\|_{\infty}$ \\
\hline 3.0 & $4.0 \mathrm{E}-4$ & 0.66 & 0.0014 & 3.57 & 0.00079 \\
2.0 & $4.3 \mathrm{E}-4$ & 0.81 & 0.0030 & 3.35 & 0.00030 \\
1.0 & $9.1 \mathrm{E}-5$ & 1.09 & 0.0074 & 3.07 & 0.00016 \\
0.0 & $-2.8 \mathrm{E}-3$ & 1.89 & 0.024 & 2.66 & 0.00088 \\
-1.0 & $-6.7 \mathrm{E}-2$ & 12.75 & 0.13 & 1.72 & 0.0159 \\
-2.0 & $-3.6 \mathrm{E}-2$ & 8.71 & 0.15 & 0.72 & 0.0089 \\
-2.75 & $-6.9 \mathrm{E}-2$ & 10.73 & 0.27 & 0.38 & 0.020 \\
\hline
\end{tabular}


A few comments are in order. The reason for defining the "overlap" and "harmonic dominance" parameters $\mu$ and $\nu$ is that when the amplitude $\alpha$ is large, the soliton overlap is small $(\mu \ll 1)$ and each soliton is, to a first approximation, an independent solution of the quartic $K d V$ which does not interact with any of its copies. It follows that soliton-superposition is trivially a good approximation in the sense that we expect an error no worse than $0(\mu \alpha)$, i.e., no worse than amplitude of two neighboring solitons at the point of maximum overlap, for arbitrary nonlinear wave equations.

What is remarkable about Table 1 is that it shows that the error is much smaller than expected for a very wide range of amplitudes. When $c_{\text {sol }}=3$, the magnitude of $S(x)$ and $S(x+2 \pi)$ at $x=\pi$, their point of maximum overlap, is $1 / 200$, but the maximum difference between the imbricate-soliton series and the exact hypercniodal wave is only $1 / 1265$.

As $c$ decreases, the amplitude $\alpha \rightarrow 0$. The "harmonic dominance" parameter shows that, although the progression is not monotonic, the Fourier series of the hypercnoidal wave is more dominated by the lowest harmonic. In other words, the small amplitude hypercnoidal wave differs by less than $10 \%$ (for the last three entries in the table) from a cosine wave. Even in this regime, however, the maximum error in the imbricate-soliton approximation is still small in comparison to the overlap between neighboring solitons.

\section{Summary \& Open Problems}

The principal conclusions are that (i) the approximation

hypercnoidal wave $\approx$ imbricate-soliton series

is more accurate than one had any right to expect and (ii) the soliton-superposition theorems cannot be extended to the quartic Korteweg-deVries equation because (11) is only an approximation. Several open problems remain. The first is to understand why soliton-superposition is exact for so many wave equations and such a remarkably good approximation for the quartic $K d V$ equation. The theorem-provers might say with $\mathrm{Dr}$. Sam Johnson: "I have given you, Sir, a reason; I am not required to give you an explanation". Science, however, is about explanations, and at the moment, we have only proofs.

The Korteweg-deVries and Kadomtsev-Petviashvili equations have multiply-periodic solutions known as " $N$-polycnoidal" waves (among other names [11]). The $K d V$ polycnoidal waves generalize the $N$-soliton solution (for $N>1$ ) in the same way that the ordinary cnoidal wave generalizes the soliton; they are known to be hyperelliptic functions. It is natural to ask: are the polycnoidal waves the exact imbrication of the $N$-solitons? The failure of exact soliton-superposition for the hyperelliptic solutions of the quartic $K d V$ suggests that the imbricate series will fail for the $K d V$ polycnoidal waves, too-but as of now, this is an untested conjecture.

The third open problem is to find an example of a non-elliptic cnoidal wave which is the exact superposition of solitons or a proof that none exists.

Lastly, the large amplitude, solitary wave regime and the small amplitude, linear cosine wave dynamics have considerable overlap, and periodic solutions of intermediate amplitude may be regarded as either solitons or sine waves with little error (Fig. 1) -for the quartic $K d V$, for the non-integrable equation of [7], and for all those equations for which soliton-superposition is exact. The fourth open problem is to apply imbricate series of solitary waves to other problems as an analytical tool for exploring this intriguing soliton/sine wave duality. 


\title{
References
}

[1] M. Toda, Physics Rep, 18, 1, (1975).

[2] J. P. Boyd, SIAM J. Appl. Math. 44, 952 (1984).

[3] A. A. Zaitsev, Sov. Phys. Dokl. 28, 720 (1983).

[4] A. Korpel and P. P. Banerjee, Phys. Lett. 82A, 113 (1981).

[5] G. B. Whitham, IMA J. Appl. Math. 32, 353 (1984).

[6] B. Fornberg and G. B. Whitham, Phil. Trans. Roy. Soc. London 289, 32 (1978).

[7] J. P. Boyd, Physica 21D, 227 (1986).

[8] H. E. Rauch and H. M. Farkas, Theta Functions with Applications to Riemann Surfaces. Williams \& Wilkins, Baltimore 1974.

[9] J. P. Boyd, J. Comp. Phys. 69, 112 (1986).

[10] J. L. Bona, W. G. Pritchard and L. R. Scott, Phys. Fluids 23, 438 (1980).

[II] J. P. Boyd, J, Math. Phys. 25, 3390 (1984).

[12] J. P. Boyd, Periodic Solutions Generated by Solitons for the Quartically Nonlinear Korteweg-deVries Equation. Technical Rept. 88-4, Laboratory for Scientific Computation, College of Engineering, University of Michigan, $20 \mathrm{pp}$.

\begin{abstract}
Toda [1], Boyd [2], Zaitsev [3], Korpel \& Banerjee [4], and Whitham [5] have proved that many species of solitons may be cloned and superposed with even spacing to generate exact nonlinear, spatially periodic solutions ("cnoidal waves"). The equations solved by such "imbricate" series of solitary waves include the Korteweg-deVries, Cubic Schroedinger, Benjamin-Ono, and resonant triad equations. However, all existing theorems apply only when the solitons are rational or meromorphic functions and the cnoidal waves are elliptic functions. In this note, we ask: does the exact soliton-superposition apply to non-elliptic solitons and cnoidal waves?

Although a complete answer to this (very broad!) question eludes us, it is possible to offer a revealing counterexample. The quartic Korteweg-deVries equation has solutions which are hyperelliptic, and thus very special. Nevertheless, its periodic solutions are not the exact superposition of the infinite number of copies of a soliton. This is highly suggestive that non-elliptic extensions of the Toda theorem are rare or non-existent. It is intriguing, however, that the soliton-superposition generates a very good approximation to the hypercnoidal wave even when the solitons strongly overlap.
\end{abstract}

(Received: September 7, 1988; revised: July 4, 1989) 